\title{
Tight junctions in thyroid carcinogenesis: diverse expression of claudin-1, claudin-4, claudin-7 and occludin in thyroid neoplasms
}

\author{
Vassiliki N Tzelepi ${ }^{1}$, Athanassios C Tsamandas ${ }^{1}$, Helen D Vlotinou ${ }^{1}$, \\ Constantine E Vagianos ${ }^{2}$ and Chrisoula D Scopa ${ }^{1}$ \\ ${ }^{1}$ Faculty of Medicine, Department of Pathology, University Hospital, University of Patras, Patras, Greece and \\ ${ }^{2}$ Faculty of Medicine, Department of Surgery, University Hospital, University of Patras, Patras, Greece
}

\begin{abstract}
Claudins and occludin are integral constituents of tight junctions and are deregulated in a variety of malignancies. Their role in thyroid carcinogenesis has not yet been elucidated. This study investigates the expression of occludin and claudin-1, -4 and -7 in thyroid neoplasms. Ninety-one thyroid neoplasms (15 follicular adenomas, 15 follicular carcinomas, 26 papillary carcinomas, 16 papillary microcarcinomas, 8 medullary carcinomas, 3 poorly differentiated carcinomas, 8 undifferentiated carcinomas) were immunostained with antibodies against occludin and claudin-1, -4 and -7 . Occludin was mainly expressed in the form of intracytoplasmic vesicles, whereas all claudins tested exhibited membranous immunostaining. Thirteen out of 15 follicular adenomas, 10/15 follicular carcinomas, 24/26 papillary carcinomas, 15/16 papillary microcarcinomas, 1/8 medullary carcinomas, 2/3 poorly differentiated carcinomas and 2/8 undifferentiated carcinomas exhibited claudin-1 expression, whereas claudin-4 was expressed in 13/15, 12/15, 23/26, 13/16, 7/8, 2/3 and 2/8 of the tumors, respectively, and claudin-7 expression was found in $67,33,73,69,25,0$ and $13 \%$ of the cases, respectively. Occludin was expressed in $100 \%$ follicular adenomas, $80 \%$ follicular carcinomas, $96 \%$ papillary carcinomas, $50 \%$ papillary microcarcinomas, $50 \%$ medullary carcinomas, $33 \%$ poorly differentiated carcinomas and $\mathbf{8 8} \%$ undifferentiated carcinomas. Occludin expression was reduced in papillary microcarcinomas, medullary carcinomas and poorly differentiated carcinomas. All claudins exhibited reduced expression in undifferentiated carcinomas. Claudin-1 was additionally reduced in medullary carcinomas and claudin-7 in follicular, medullary and poorly differentiated carcinomas. A correlation between loss of claudin-1 expression and worse disease-free survival was noted on univariate analysis. Dedifferentiation of the thyroid carcinomas is accompanied by reduction in claudin-1, -4 and -7 expression. A differential expression of tight junction proteins in the different histologic types of thyroid gland is noted. Additionally, claudin-1 expression may be an important prognostic indicator of recurrence in thyroid carcinomas.
\end{abstract}

Modern Pathology (2008) 21, 22-30; doi:10.1038/modpathol.3800959; published online 26 October 2007

Keywords: claudins; differentiation; occludin; thyroid tumors; tight junctions

Tight junctions are intercellular junctional complexes located at the apical end of the lateral membranous surface of polarized cells. Their main role is the prevention of free diffusion of solutes through paracellular spaces, known as the barrier function. In addition, tight junctions block the free diffusion of proteins and lipids between the apical and basolateral domains of the plasma membrane, therefore maintaining cell polarity (fence function). ${ }^{1,2}$

Correspondence: Dr CD Scopa, MD, Department of Pathology, University of Patras, School of Medicine, PO Box 1174, Patras 261 10, Greece.

E-mail: cdscopa@med.upatras.gr

Received 15 March 2007; revised 9 August 2007; accepted 10 August 2007; published online 26 October 2007
Tight junctions are composed of transmembrane and cytoplasmic protein complexes and appear on electron micrographs as series of fusion points between the outer leaflets of plasma membranes of adjacent cells. At these so-called 'kissing points', the intercellular space is eliminated remarkably., ${ }^{2,3}$ Up to now, three groups of macromolecules are considered as bona fide integral components of tight junctions: occludin, claudins and junctional adhesion molecule. ${ }^{2,4}$

Occludin represents the first transmembrane protein of tight junctions that was identified and is a $65 \mathrm{kDa}$ protein that spans the membrane four times, thus having two extracellular loops. ${ }^{5}$ The cytoplasmic $\mathrm{N}$ - and C-terminal domains contain several phosphorylation sites and interact with various proteins. ${ }^{1,2}$ The exact role of occludin in 
tight junctions function is unclear, although a regulatory function is speculated. ${ }^{1}$

Claudins are integral membrane proteins that have, similar to occludin, four transmembrane domains and two extracellular loops. ${ }^{6,7}$ However, unlike occludin, the first loop is longer than the second one. ${ }^{8}$ Claudins were named after the latin verb 'claudere', which means 'to close', reflecting their role in tight junctions: they are the key proteins for the sealing of the extracellular space. ${ }^{7-10}$ Claudin family consists of 24 members. Different claudins are found at the tight junction of diverse epithelial cells, ${ }^{11}$ and this accounts for the observed differences in permeability and electrical resistance of various epithelia. ${ }^{2,8,10}$

Previous studies have shown that occludin and claudin-1, -4 and -7 are deregulated in a variety of malignancies; for example, loss of occludin expression or upregulation of claudin- 3 and -4 accompanies the progression of endometrial carcinoma, ${ }^{12,13}$ reduced expression of claudin-1 correlates with poorer differentiation, disease recurrence and poor survival in stage II colonic cancer, ${ }^{14}$ claudin-7 expression has been found reduced in mammary, ${ }^{15}$ esophageal, ${ }^{16}$ and head and neck carcinomas, ${ }^{17}$ whereas claudin-4 overexpression decreases the invasiveness and metastatic potential of pancreatic cancer cells. ${ }^{18}$ On the other hand, various cancers (ie ovarian, colon, cervical cancer) are associated with elevated tight junctions proteins expression levels or protein dislocation to the cytoplasm. ${ }^{19-21}$ These reports emphasize the complex and diverse actions of tight junctions proteins in cancer progression.

Follicular cells of the thyroid gland are arranged in a single highly polarized layer and act as a barrier between the lumen of the follicle, where thyroglobulin and thyroid hormones are stored, and the extrafollicular space. Epithelial cell polarity and follicular space entrenchment are due to the presence of firm tight junctions. In a recent study, elevated gene expression of several claudins (ie claudin-3, -4, -7) was found in normal and malignant thyroid tissue. ${ }^{22}$ However, the expression of tight junctions proteins in the various histologic types of thyroid neoplasms has not been addressed thoroughly before.

This study investigates the expression of occludin and the claudin-1, -4 and -7 in various thyroid neoplasms and in the adjacent non-neoplastic thyroid tissue by immunohistochemistry, in an attempt to clarify the relationship between tight junctions' protein expression and tumor type.

\section{Materials and methods}

Formalin-fixed, paraffin-embedded thyroid tissues of 91 thyroidectomy specimens resected from an equal number of patients for therapeutic purposes, as well as the pathology report for each patient, were retrieved from the files of the Department of
Pathology, University Hospital of Patras, Greece. There were 19 males and 72 females, ranging from 19 to 84 years old (mean: $47 \pm 14$ ). All available slides were reviewed and the most representative block from each case was selected. Each case was classified according to the WHO histologic classification of thyroid tumors. ${ }^{23}$ The material consisted of 15 cases of follicular adenomas, 15 follicular carcinomas, 26 papillary carcinomas, 16 papillary microcarcinomas, 8 medullary carcinomas, 3 poorly differentiated carcinomas and 8 undifferentiated carcinomas. For each tumor, several features were evaluated and finally the tumor size and stage $(\mathrm{T})^{24}$ were recorded. Additionally, follow-up data were available for 33 patients (1 patient with papillary microcarcinoma, 13 with papillary carcinoma, 7 with medullary carcinoma, 2 with undifferentiated carcinoma and 10 with follicular carcinoma) and the disease-free survival and overall survival was determined.

\section{Immunohistochemistry}

Immunohistochemistry was performed on $4-\mu \mathrm{m}$-thick formalin-fixed, paraffin-embedded tissue sections mounted on gelatin-coated glass slides. Deparaffination, rehydration and antigen retrieval were performed in an electric pressure cooker using Trilogy retrieval solution (Cell Marque, AR, USA) for $30 \mathrm{~min}$. Polyclonal antibodies against occludin (1:80), claudin-1 (1:100) and claudin-7 (1:200) and monoclonal antibodies against claudin-4 (3E2C1, 1:100) were used for the primary reaction. All antibodies were purchased from Zymed (CA, USA). The sections were incubated with primary antibodies for 1 (occludin and claudin-1) or 2 (claudin-4 and -7) hours at room temperature, followed by sequential 30 min incubation with Dako EnVision Labelled Polymer (Dako, CA, USA). Diaminobenzidine (Dako) was used as the chromogen. Nuclei were counterstained with Harris hematoxylin. Sections from breast carcinoma were used as positive control for occludin. Normal skin was the positive control for claudin-1 and normal colon for claudin-4 and -7. In negative control slides, the same method was performed and the primary antibody was substituted by $1 \%$ TBS. Membranous and cytoplasmic immunostaining of occludin was considered positive. For all antibodies, cases exhibiting $>5 \%$ positive cells were considered as positive. ${ }^{25,26}$ The adjacent non-neoplastic thyroid tissue was also evaluated.

\section{Statistical Analysis}

Results were expressed as number of positive cases per number of total cases. The $\chi^{2}$-test was used to record any differences between the staining results and the tumor features. The analysis of overall survival and disease-free survival was calculated with the Kaplan-Meier method and the differences in survival between the groups were compared 
using the log-rank test. Multivariate analysis including clinicopathologic factors and expression of each protein was performed using Cox proportional hazards method. All data were analyzed with the SPSS program $\left(\right.$ SPSS $^{\circledR}$ 14.0, Chicago, USA). Any $P$ value $<0.05$ was considered as significant.

\section{Results}

\section{Routine Pathology}

Tumor size ranged from 0.3 to $9 \mathrm{~cm}(2.4 \pm 1.9 \mathrm{~cm})$ in greatest diameter. Three tumors exhibited oncocytic differentiation. They were two follicular adenomas (Hürthle cell adenoma) and one papillary carcinoma (oncocytic variant) and were all noted in women with a mean age of $41 \pm 16$ years.

Regarding tumor stage, ${ }^{24} 32$ tumors were $\mathrm{T} 1$, 14 T2, 21 T3 and 9 T4. In 13 cases, lymph node sampling had been performed. Six of them were No (two follicular carcinomas, three papillary carcino-

Table 1 Tumor stage and size of the 88 thyroid neoplasms

\begin{tabular}{lrrrrr}
\hline Histologic type & \multicolumn{3}{c}{ Pathologic parameter } \\
\hline Stage & T1 & T2 & T3 & T4 \\
Papillary microcarcinoma $(N=16)$ & 16 & - & - & - \\
Papillary carcinoma $(N=26)$ & 10 & 4 & 12 & - \\
Follicular carcinoma $(N=15)$ & 4 & 6 & 5 & - \\
Medullary carcinoma $(N=8)$ & 2 & 3 & 3 & -1 \\
Poorly differentiated & - & 1 & 1 & 1 \\
carcinoma $(N=3)$ & & & & \\
Undifferentiated & - & - & - & 8 \\
carcinoma $(N=8)$ & & & & \\
Size (cm) & $1-2$ & $2-4$ & $>4$ & Unknown \\
Follicular adenoma $(N=15)$ & 10 & 4 & 1 & -2 \\
Papillary carcinoma $(N=26)$ & 13 & 6 & 5 & 2 \\
Follicular carcinoma $(N=15)$ & 4 & 5 & 5 & 1 \\
Medullary carcinoma $(N=8)$ & 4 & 1 & 1 & 2 \\
Poorly differentiated & - & 1 & - & 2 \\
carcinoma $(N=3)$ & & & & \\
Lymph node status & No & N1 & & & \\
Papillary microcarcinoma $(N=1)$ & 1 & - & & \\
Papillary carcinoma $(N=6)$ & 3 & 3 & & \\
Medullary carcinoma $(N=4)$ & 0 & 4 & & \\
\hline
\end{tabular}

$\mathrm{T}$, tumor stage. mas and one papillary microcarcinoma), whereas the remaining 7 (three papillary and four medullary carcinomas) were N1. Table 1 lists the distribution of the different histologic types according to the tumor stage, tumor size and lymph node status.

Follow-up data were available for 33 of the 76 (43\%) patients with malignant thyroid neoplasms (including papillary microcarcinomas) with a mean follow-up period of $60 \pm 47$ months (range 1-144 months). During this period of time, two deaths (one patient with medullary and one with undifferentiated carcinoma) and six relapses (one patient with papillary carcinoma, four patients with medullary and one with undifferentiated carcinoma) were recorded.

\section{Immunohistochemistry}

\section{Claudins expression}

In general, positive claudin immunostaining was observed in $91 \%$ (83/91) of the cases. Claudins expression in the different histologic types is shown in Table 2. All claudins examined were expressed at the basolateral membrane surfaces in a linear fashion. However, a membranous dot-like pattern was noted quite often and occasionally the immunostaining was in the form of small vesicles located at the membrane. Although dots and vesicles could be found at any part of the basolateral membrane, they were more commonly seen at the apical end of the lateral membrane surface (Figure 1). In nonneoplastic thyroid tissue, claudins were focally expressed, mainly at hyperplastic follicular cells.

\section{Claudin-1}

Claudin-1 (Figure 1a) expression was present in $74 \%(67 / 91)$ of the cases, and more specifically in $87 \%$ of the follicular adenomas, $67 \%$ of the follicular carcinomas, $92 \%$ of the papillary carcinomas, $93 \%$ of the papillary microcarcinomas, $13 \%$ of the medullary carcinomas, $67 \%$ of the poorly differentiated carcinomas and $25 \%$ of the undifferentiated carcinomas. Statistical analysis revealed a significant reduction of claudin-1 expression in medullary and undifferentiated carcinomas compared to the other histologic types $(P<0.001)$.

Table 2 Claudins' and occludin's expression in thyroid neoplasms

\begin{tabular}{|c|c|c|c|c|c|}
\hline & Claudin-1 & Claudin-4 & Claudin-7 & Occludin & $\mathrm{P}$ \\
\hline Follicular adenoma $(N=15)$ & $13^{\mathrm{a}}(87 \%)$ & $13(87 \%)$ & $10(67 \%)$ & $15(100 \%)$ & 0.083 \\
\hline Follicular carcinoma $(N=15)$ & $10(67 \%)$ & $12(80 \%)$ & $5(33 \%)$ & $12(80 \%)$ & $\mathbf{0 . 0 0 7}$ \\
\hline Papillary microcarcinoma $(N=16)$ & $15(93 \%)$ & $13(81 \%)$ & $11(69 \%)$ & $8(50 \%)$ & 0.036 \\
\hline Papillary carcinoma $(N=26)$ & $24(92 \%)$ & $23(88 \%)$ & $19(73 \%)$ & $25(96 \%)$ & 0.063 \\
\hline Medullary carcinoma $(N=8)$ & $1(12.5 \%)$ & $7(87.5 \%)$ & $2(25 \%)$ & $4(50 \%)$ & 0.014 \\
\hline Poorly differentiated carcinoma $(N=3)$ & $2(67 \%)$ & $2(67 \%)$ & $0(0 \%)$ & $1(33 \%)$ & 0.287 \\
\hline Undifferentiated carcinoma $(N=8)$ & $2(25 \%)$ & $2(25 \%)$ & $1(13 \%)$ & $7(88 \%)$ & 0.008 \\
\hline$P$-value & $<\mathbf{0 . 0 0 1}$ & 0.01 & 0.002 & $<\mathbf{0 . 0 0 1}$ & \\
\hline
\end{tabular}

Bold typing indicate $P$-values $<0.05$.

${ }^{\mathrm{a}}$ No. of positive cases. 

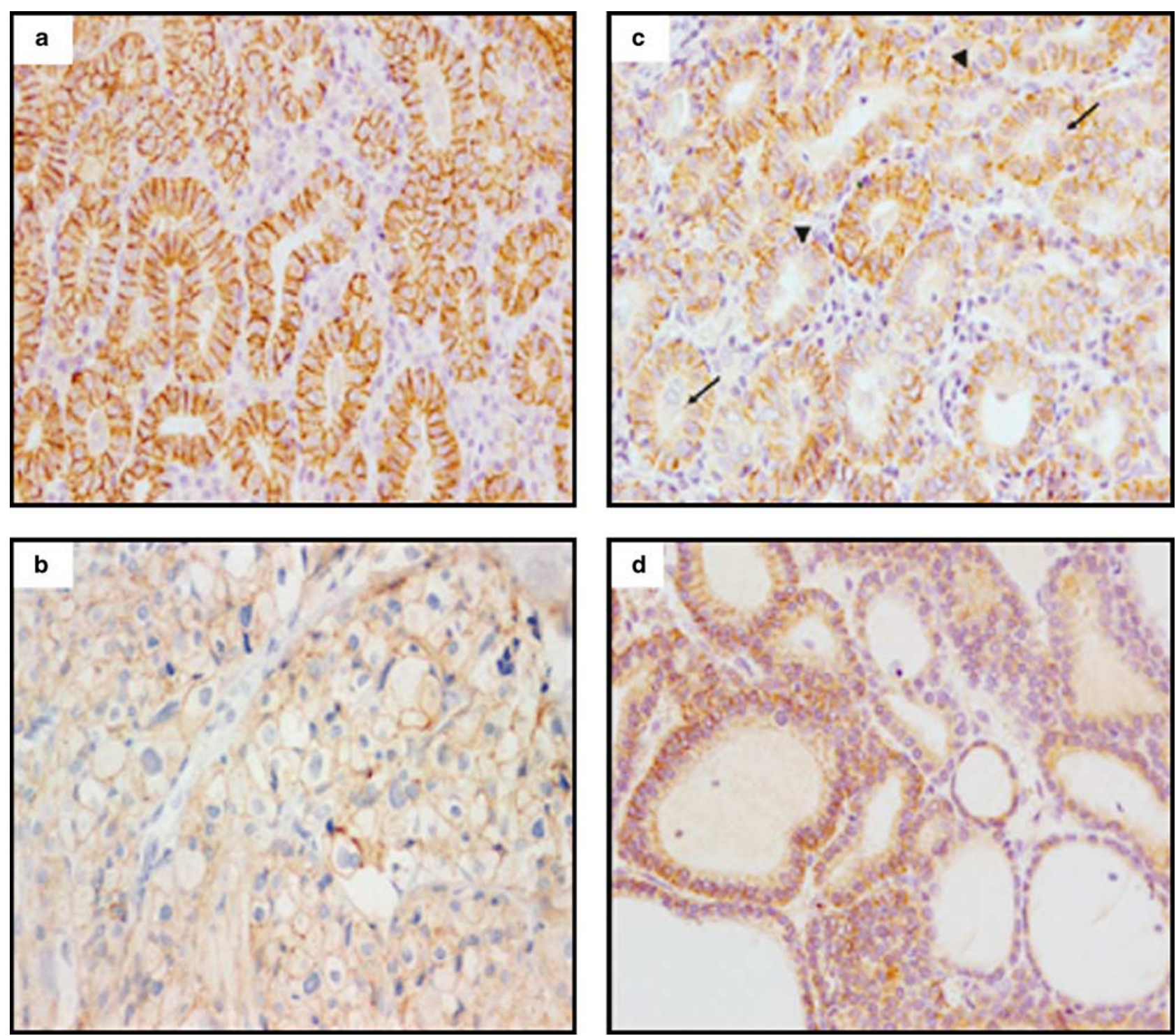

Figure 1 Immunohistochemical analysis of claudins' expression in thyroid neoplasms (streptavidin biotin peroxidase, $\times 400)$. (a) Strong membranous immunostaining of claudin-1 in a case of papillary carcinoma. (b) Cells of medullary carcinoma express claudin-4 in their membranes. (c) Continuous and dot-like membranous immunostaining of claudin-7 in papillary carcinoma. Dots are located at the apical end of the lateral membranous surface (arrow) and other parts of the lateral and basal membranous surface as well (arrowhead). (d) Continuous and dot-like immunostaining of claudin-7 in a case of follicular carcinoma.

\section{Claudin-4}

Claudin-4-positive immunostaining (Figure 1b) was observed in 72 of the 91 cases examined $(79 \%)$ and in particular in $87 \%$ follicular adenomas, $80 \%$ follicular carcinomas, $88 \%$ papillary carcinomas, $81 \%$ papillary microcarcinomas, $88 \%$ medullary carcinomas, $67 \%$ poorly differentiated carcinomas and $25 \%$ undifferentiated carcinomas. Claudin-4 expression was significantly reduced in undifferentiated carcinomas compared to the other histologic types $(P=0.01)$.

\section{Claudin-7}

Claudin-7 (Figure $1 \mathrm{c}$ and d) was expressed in $53 \%(48 / 91)$ of the tumors. Claudin-7 expression was found in $67 \%$ of the follicular adenomas, $33 \%$ of the follicular carcinomas, $73 \%$ of the papillary carcinomas, $69 \%$ of the papillary microcarcinomas, $25 \%$ of the medullary carcinomas, $0 \%$ of the poorly differentiated carcinomas and $13 \%$ of the undifferentiated carcinomas. Statistical analysis revealed that claudin-7 was less frequently expressed in follicular, medullary, poorly differentiated and undifferentiated carcinomas as opposed to papillary carcinomas, papillary microcarcinomas and adenomas $(P=0.002)$.

\section{Occludin}

Occludin was expressed in 72 of the 91 (79\%) cases examined. Occludin's expression in the thyroid tumors examined is shown in Table 2. Of interest, occludin, although a tight junction protein, was only 
rarely detected at the membranous surface (17 of the 72 positive cases). In the vast majority of the cases, occludin was detected as intracytoplasmic vesicles either alone (55 of the 72 positive cases) or together with membranous staining (10 cases: three adenomas, three papillary microcarcinomas, two papillary carcinomas, one medullary carcinoma and one undifferentiated carcinoma) (Figure 2) and only in seven cases (three adenomas, two papillary microcarcinomas, one papillary carcinoma and one poorly differentiated carcinoma) exhibited pure membranous staining pattern. In particular, occludin expression was noted in $100 \%$ of the follicular adenomas, in $80 \%$ of the follicular carcinomas, $96 \%$ of the papillary carcinomas, $50 \%$ of the papillary microcarcinomas, $50 \%$ of the medullary carcinomas, $33 \%$ of the poorly differentiated carcinomas and $88 \%$ of the undifferentiated carcinomas. Statistical analysis revealed a reduction of occludin expression in papillary microcarcinomas, poorly differentiated carcinomas and medullary carcinomas compared to the other histologic types of thyroid tumors $(P<0.001)$. In non-neoplastic thyroid tissue occludin was focally expressed, mainly at hyperplastic follicular cells.

\section{Statistical Analysis}

Tight junctions' proteins expression was further correlated with histologic type, tumor size and stage and lymph node status.

Claudin-1 was not expressed in T2 medullary carcinomas in contrast to papillary and follicular carcinomas of the same stage $(P=0.004)$. The same applied for occludin expression. A reduction of claudin-1 expression was also noted in T3 medullary and follicular carcinomas compared to T3 papillary carcinomas $(P=0.010$ and 0.011 , respectively) (Table 3).
Claudin-4 was the most frequently expressed tight junction protein in medullary carcinomas $(P=0.014)$ (Table 2). Moreover, follicular carcinomas greater than $4 \mathrm{~cm}$ expressed more frequently claudin-4 and occludin than claudin-1 and 7 $(P=0.04)$ (Table 4). The same was true for follicular carcinomas stage T3 $(P=0.04)$ (Table 3).

Claudin-7 expression was reduced in follicular carcinomas compared to the other proteins tested $(P=0.007)$. Moreover, papillary carcinomas expressed less commonly claudin-7, compared to the other claudins and occludin, although the difference did not reach statistical significance $(P=0.063)$ (Table 2).

Occludin was the only tight junction protein frequently expressed in undifferentiated carcinomas $(P=0.008)$. The opposite was true for papillary microcarcinomas $(P=0.036)$ (Table 2). Regarding tumor size, papillary carcinomas measuring less than $1 \mathrm{~cm}$ (microcarcinomas) exhibited reduced expression of occludin compared to papillary carcinomas of size $2-4 \mathrm{~cm}(P=0.021)$. Surprisingly, occludin expression was also reduced in tumors measuring $>4 \mathrm{~cm}$ (Table 4 ).

No correlation between claudins and occludin expression and lymph node status was noted.

\section{Univariate and multivariate survival analysis}

On univariate analysis, tumor type (medullary and undifferentiated carcinomas compared to the other neoplasms) and loss of claudin-1 expression correlated with worse disease-free survival $(P=0.01)$ (Figure 3$)$.

Tumor type and stage were correlated with overall survival $(P=0.02$ and 0.002 , respectively). Additionally, increased expression of claudin-1 was
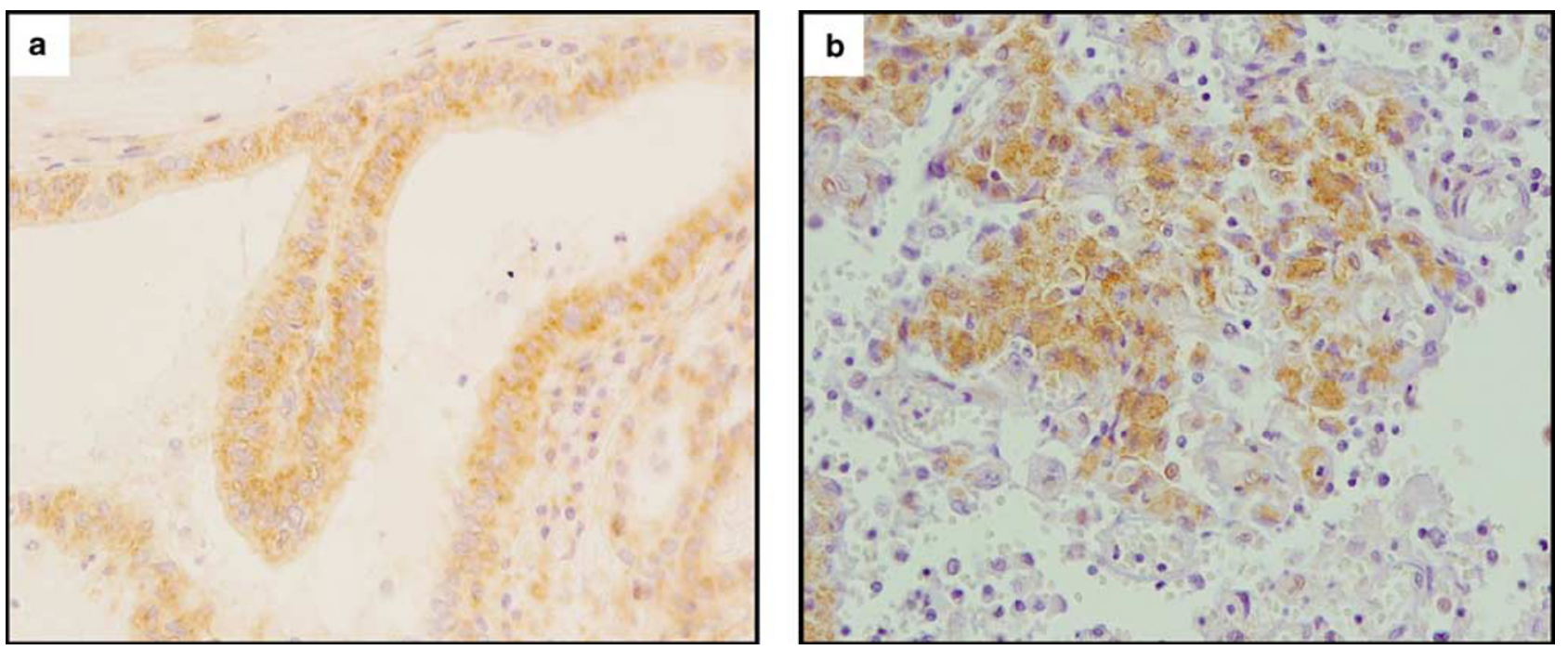

Figure 2 Immunohistochemical expression of occludin in a case of papillary (a) and undifferentiated (b) carcinoma (streptavidin biotin peroxidase, $\times 400$ ). 
Table 3 Correlation between tumor's stage and claudins' and occludin's expression for papillary carcinomas, follicular carcinomas, medullary carcinomas, poorly differentiated carcinomas and undifferentiated carcinomas

\begin{tabular}{|c|c|c|c|c|c|}
\hline & Stage $(T)$ & Claudin-1 & Claudin-4 & Claudin-7 & Occludin \\
\hline \multirow[t]{3}{*}{ Papillary carcinoma } & $\mathrm{T} 1(N=10)$ & $9^{\mathrm{a}}(90 \%)$ & $8(80 \%)$ & $8(80 \%)$ & $10(100 \%)$ \\
\hline & $\mathrm{T} 2(N=4)$ & $4(100 \%)$ & $3(75 \%)$ & $3(75 \%)$ & $4(100 \%)$ \\
\hline & T3 $(N=12)$ & $11(92 \%)$ & $12(100 \%)$ & $8(67 \%)$ & $11(92 \%)$ \\
\hline \multirow[t]{3}{*}{ Follicular carcinoma } & $\mathrm{T} 1(N=4)$ & $3(75 \%)$ & $2(50 \%)$ & $1(25 \%)$ & $3(75 \%)$ \\
\hline & $\mathrm{T} 2(N=6)$ & $5(83 \%)$ & $4(67 \%)$ & $3(50 \%)$ & $5(83 \%)$ \\
\hline & $\mathrm{T} 3(N=5)$ & $2(40 \%)$ & $5(100 \%)$ & $1(20 \%)$ & $4(80 \%)^{\mathrm{b}}$ \\
\hline \multirow[t]{3}{*}{ Medullary carcinoma } & $\mathrm{T} 1(N=2)$ & $1(50 \%)$ & $2(100 \%)$ & $1(50 \%)$ & $2(100 \%)$ \\
\hline & $\mathrm{T} 2(N=3)$ & $0(0 \%)$ & $2(66.7 \%)$ & $0(0 \%)$ & $0(0 \%)$ \\
\hline & T3 $(N=3)$ & $0(0 \%)$ & $1(33 \%)$ & $0(0 \%)$ & $2(67 \%)$ \\
\hline \multirow[t]{4}{*}{ Poorly differentiated carcinoma } & $\mathrm{T} 1(N=0)$ & - & - & - & - \\
\hline & $\mathrm{T} 2(N=1)$ & $1(100 \%)$ & $1(100 \%)$ & $0(0 \%)$ & $0(0 \%)$ \\
\hline & T3 $(N=1)$ & $0(0 \%)$ & $0(0 \%)$ & $0(0 \%)$ & $0(0 \%)$ \\
\hline & $\mathrm{T} 4(N=1)$ & $1(1000 \%)$ & $1(100 \%)$ & $0(0 \%)$ & $1(100 \%)$ \\
\hline \multirow[t]{2}{*}{ Undifferentiated carcinoma } & $\mathrm{T} 1 / \mathrm{T} 2 / \mathrm{T} 3(N=0)$ & - & - & - & - \\
\hline & $\mathrm{T} 4(N=8)$ & $2(25 \%)$ & $2(25 \%)$ & $1(123 \%)$ & $7(88 \%)$ \\
\hline
\end{tabular}

${ }^{\mathrm{a}}$ No. of positive cases.

${ }^{\mathrm{b}} P=0.04$.

Table 4 Correlation between tumor's size and claudins' and occludin's expression for papillary carcinomas (including microcarcinomas), follicular adenomas, follicular carcinomas and medullary carcinomas

\begin{tabular}{|c|c|c|c|c|c|}
\hline & Size $(\mathrm{cm})$ & Claudin-1 & Claudin-4 & Claudin-7 & Occludin \\
\hline Papillary carcinoma and microcarcinoma & $\begin{array}{l}<1(N=16) \\
1-2(N=13) \\
2-4(N=6) \\
>4(N=5)\end{array}$ & $\begin{array}{c}15^{\mathrm{a}}(94 \%) \\
13(100 \%) \\
5(83 \%) \\
3(60 \%)\end{array}$ & $\begin{array}{r}13(81 \%) \\
12(92 \%) \\
4(67 \%) \\
4(80 \%)\end{array}$ & $\begin{array}{r}11(69 \%) \\
11(85 \%) \\
4(67 \%) \\
2(40 \%)\end{array}$ & $\begin{array}{c}8(50 \%)^{\mathrm{b}} \\
13(100 \%) \\
5(83 \%) \\
2(40 \%)\end{array}$ \\
\hline Follicular adenoma & $\begin{array}{c}<1(N=0) \\
1-2(N=10) \\
2-4(N=4) \\
>4(N=1)\end{array}$ & $\begin{array}{l}-\overline{(90 \%)} \\
3(75 \%) \\
1(100 \%)\end{array}$ & $\begin{array}{l}-\overline{(90 \%)} \\
3(75 \%) \\
1(100 \%)\end{array}$ & $\begin{array}{l}-\overline{1}(10 \%) \\
3(75 \%) \\
1(100 \%)\end{array}$ & $\begin{array}{r}-\overline{(100 \%)} \\
4(100 \%) \\
1(100 \%)\end{array}$ \\
\hline Follicular carcinoma & $\begin{array}{r}<1(N=0) \\
1-2(N=4) \\
2-4(N=5) \\
>4(N=5)\end{array}$ & $\begin{array}{l}3(75 \%) \\
5(100 \%) \\
2(40 \%)\end{array}$ & $\begin{array}{l}-\overline{-} \\
2(50 \%) \\
4(80 \%) \\
5(100 \%)\end{array}$ & $\begin{array}{l}-\overline{(25 \%)} \\
3(60 \%) \\
1(20 \%)\end{array}$ & $\begin{array}{l}-\overline{(75 \%)} \\
5(100 \%) \\
4(80 \%)^{\mathrm{c}}\end{array}$ \\
\hline Medullary carcinomas & $\begin{array}{r}1-2(N=4) \\
2-4(N=1) \\
>4(N=1)\end{array}$ & $\begin{array}{l}1(25 \%) \\
0(0 \%) \\
0(0 \%)\end{array}$ & $\begin{array}{l}3(75 \%) \\
1(100 \%) \\
1(100 \%)\end{array}$ & $\begin{array}{l}1(25 \%) \\
0(0 \%) \\
1(100 \%)\end{array}$ & $\begin{array}{l}2(50 \%) \\
0(0 \%) \\
1(100 \%)\end{array}$ \\
\hline Poorly differentiated carcinoma & $\begin{array}{r}<2(N=0) \\
2-4(N=1) \\
>4(N=0)\end{array}$ & $1 \overline{(100 \%)}$ & $1(\overline{(100 \%)}$ & - & $\begin{array}{l}- \\
-\end{array}$ \\
\hline
\end{tabular}

${ }^{\mathrm{a}}$ No. of positive cases.

${ }^{\mathrm{b}} P=0.021$.

${ }^{\mathrm{c}} P=0.04$.

associated with slightly better prognosis, although this was not statistically significant $(P=0.093)$.

Cox multivariate analysis of survival revealed that claudins and occludin's expression and the clinicopathologic parameters studied were not independently associated with disease-free or overall survival.

\section{Discussion}

This study examined the expression of tight junctions' proteins, occludin and claudin-1, -4 and -7 in thyroid neoplasms. The role of tight junctions' proteins in cancer initiation and progression has been under intense investigation. Reduced expression, 
Claudin 1

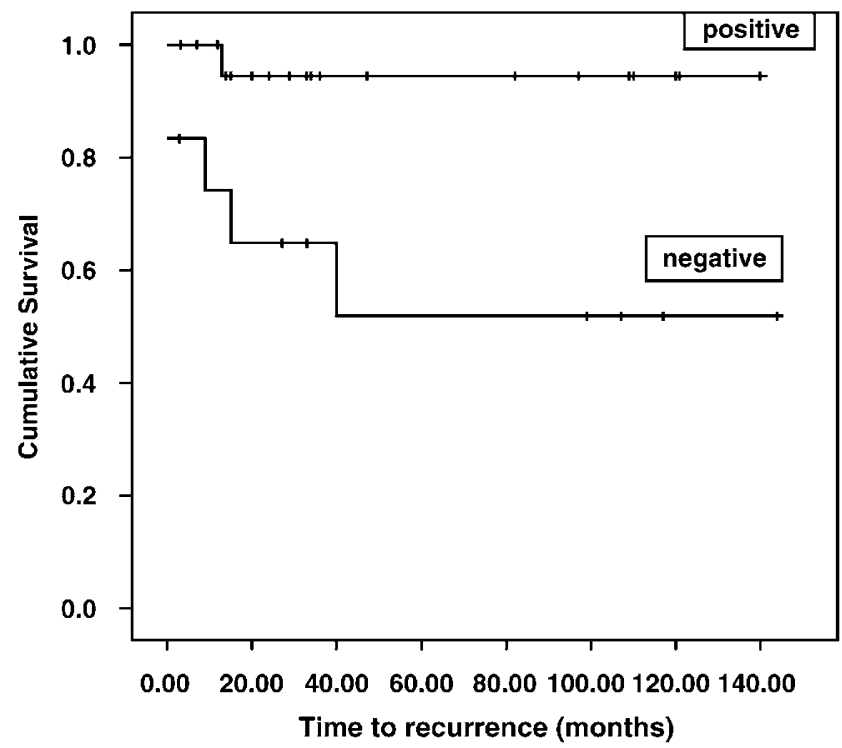

Figure 3 Kaplan-Meier disease-free analysis curve in thyroid carcinomas for claudin-1 expression. A strong association was detected between loss of protein's expression and disease recurrence $(P=0.01)$.

elevated levels or subcellular relocalization of tight junctions proteins have been reported in various human cancers and are variably associated with tumor differentiation and survival. ${ }^{13-17,19-21,27}$

Tight junctions' alterations have been observed in thyroid neoplasms by freeze-fracture analysis. ${ }^{28,29}$ In these studies, aberrant tight junction formation characterized poorly differentiated carcinomas or compact oncocytic tumors. Recently, gene expression analysis has shown enhanced expression of claudin-3, -4 and -7 genes in thyroid neoplastic tissues $^{22}$ and of claudin-1 and 16 genes in papillary carcinomas of the thyroid. ${ }^{30}$ These studies make no specific comment to the different histologic types of thyroid tumors. To the best of our knowledge, the present study is the first one to investigate the expression of tight junction proteins in the various histologic types of thyroid tumors and to correlate them with tumor stage and size and patients prognosis.

In this study, a loss of claudin-1, -4 and -7 expression was frequently seen in undifferentiated thyroid carcinomas. Loss of claudin-7 expression was also noted in poorly differentiated carcinomas. Reduced claudins' expression and associated loss of functional tight junctions are usually a feature of poorly differentiated tumors of various origins (ie breast, esophageal, gastric, colon and thyroid carcinomas). ${ }^{14-16,30,31}$ Apparently, loss of claudins expression results in loss of cell-to-cell adhesion, thereby preventing the cells to form organized epithelial structures and leading in an undifferentiated phenotype. Undifferentiated carcinomas of the thyroid gland are the result of anaplastic transformation of a pre-existing well-differentiated tumor. $^{32}$ The current study suggests that dedifferentiation of thyroid neoplasms involves tight junction impairment via downregulation of claudin-1, -4 and -7.

Claudin-4 was more frequently expressed in medullary carcinomas than the other proteins tested. Tight junctions in medullary carcinomas of the thyroid, despite their non-epithelial derivation, have been described before and are considered as an evidence of a common stem cell origin of medullary and follicular carcinomas. ${ }^{33}$ The protein composition of these tight junctions has not been illustrated so far. The present findings imply that claudin-4 is a basic constituent of tight junctions in medullary carcinomas. The significance of the expression of claudin-4 and the parallel reduced expression of the other claudins and occludin in medullary carcinomas remains unclear. Further studies with a larger series of these tumors are needed to clarify the possible roles of claudin-4 expression in medullary carcinomas and its connection with tumor growth. ${ }^{18}$

A reduction in claudin-7 expression was noted in follicular carcinomas. Moreover, claudin-7 expression, when compared to the other claudins, was found reduced in papillary carcinomas, albeit the difference did not reach statistical significance. Additionally, claudin-7 was never expressed in poorly differentiated carcinomas although no statistical significance was found possibly due to the small number of cases. This can lead to the speculation that claudin-7 loss is an early step in tight junctions protein deregulation in thyroid carcinogenesis, although further studies are needed to validate this observation.

Loss of tight junction integrity leads to an increased influx of growth factors, nutrients and other tumor-promoting molecules, thereby providing an advantage for the development, survival and growth of tumor cells. ${ }^{17,34}$ Notably, reduction of claudin-7 was more obvious in follicular carcinomas with large size or advanced stage and was accompanied by claudin-1 reduction. In contrast, T1-T2 tumors or tumors less than $4 \mathrm{~cm}$ exhibited a more uniform expression of all three claudins studied. Thus, a possible tight junction impairment in largesized or in advanced stage follicular carcinomas is suggested through claudin-1 and -7 reduction.

Occludin expression was retained in undifferentiated carcinomas of the thyroid gland. It is worth to note that occludin alone is ineffective in adequate tight junction strands formation. ${ }^{7}$ Moreover, an intracytoplasmic localization was noted in the vast majority of the tumors. The intracellular localization of occludin is difficult to explain although some speculations can be attempted, that is formation of intercellular bodies, ${ }^{35}$ tight junction remodelling ${ }^{36}$ or intracytoplasmic, tight junction unrelated function of occludin. ${ }^{37}$ An interesting finding was that occludin was less commonly expressed in papillary microcarcinomas and poorly differentiated carcinomas, compared to other histologic types. Occludin is 
considered a feature of organized epithelial structures and is commonly lost in the solid components of various cancers. ${ }^{38,39}$ These observations are not in disparity with our finding of reduced occludin expression in papillary microcarcinomas. Membranous pattern of occludin staining was more commonly seen in papillary microcarcinomas and follicular adenomas. On the contrary, papillary, medullary and undifferentiated carcinomas exhibited mainly a cytoplasmic distribution of occludin. Accordingly, poorly differentiated carcinomas displayed a reduced membranous staining $(33 \%$ of the cases). These findings may imply that occludin is located in the tight junctions of follicular adenomas and papillary microcarcinomas of the thyroid, whereas it is lost from the tight junctions of the apparently malignant forms of thyroid neoplasms.

Interestingly, claudin-1 expression was found to be a prognostic factor for disease-free survival and may prove to be a useful prognostic marker for determining the patients that are in higher risk for recurrence. In line with our observations, reduced claudin-1 expression correlates with disease recurrence and poor survival in various cancers such as colonic and prostatic carcinomas. ${ }^{14,40}$

In conclusion, to the best of our knowledge, this is the first report on claudin-1, -4, -7 and occludin expression in thyroid neoplasms. The present study revealed a differential claudin and occludin expression in the various types of thyroid neoplasms. These findings may represent histogenetic differences (epithelial vs neuroendocrine derivation) or differential impact of claudin reduction in neoplastic progression. Reduced claudins' expression correlates with loss of differentiation of thyroid cancer. Membranous occludin expression seems to correlate with thyroid neoplasms of low malignant potential, as it was frequently found positive in follicular adenomas and papillary microcarcinomas. However, the cytoplasmic distribution of this tight junction's protein in thyroid carcinomas is a challenging finding. Further research is warranted in order to elucidate the clinical relevance of aberrant tight junction expression in thyroid neoplasia and the possible prognostic implications of reduced claudin-1 expression.

\section{Acknowledgement}

We thank Drs $\mathrm{N}$ Georgopoulos, $\mathrm{K}$ Markou, $\mathrm{T}$ Alexandridis and V Kiriazopoulou from the Department of Internal Medicine, Division of Endocrinology, University Hospital, University of Patras, for providing the clinical information.

\section{References}

1 Sawada N, Murata M, Kikuchi K, et al. Tight junctions and human diseases. Med Electron Microscop 2003;36:147-156.
2 Gonzalez-Matrisal L, Bentazos A, Nava P, et al. Tight junction proteins. Prog Bio Mol Biol 2003;18:1-44.

3 Tsukita S, Furuse M. Overcoming barriers in the study of tight junctions functions: from occludin to claudin. Genes Cells 1998;3:569-573.

4 Anderson JM, Van Itallie CM. Tight junctions and the molecular basis for regulation of paracellular permeability. Am J Physiol 1995;227:G467-G475.

5 Furuse M, Hirase T, Itoh M, et al. Occludin: a novel integral membrane protein localizing at tight junctions. J Cell Biol 1993;123:1777-1788.

6 Turksen K, Troy T-C. Barriers built on claudins. J Cell Sci 2004;117:2435-2447.

7 Furuse M, Fujita K, Hiiragi T. Claudin-1 and-2: novel integral membrane proteins localizing at tight junctions with no sequence similarity to occludin. J Cell Biol 1998;141:1539-1550.

8 Mitic LL, Itallie CM, Anderson JM. Molecular physiology and pathophysiology of tight junctions. I. Tight junction structure and function: lessons from mutant animals and proteins. Am J Physiol Gastrointest Liver Physiol 2000;279:G250-G254.

9 Tsukita S, Furuse M. Pores in the wall: claudins constitute tight junction strands containing aqueous pores. J Cell Biol 2000;149:13-16.

10 Rahner C, Mitic LL, Anderson JM. Heterogeneity in expression and subcellular localization of claudins 2 , 3,4 and 5 in the rat liver, pancreas and gut. Gastroenterology 2001;120:411-422.

11 Pan XY, Wang B, Che YC, et al. Expression of claudin-3 and 4 in normal, hyperplastic, and malignant endometrial tissue. Int J Gynecol Cancer 2007;17:233-241.

12 Tobioka H, Isomura $\mathrm{H}$, Kokai $\mathrm{Y}$, et al. Occludin expression decreases with the progression of human endometrial carcinoma. Hum Pathol 2004;35:159-164.

13 Resnick MB, Konkin T, Routhier J, et al. Claudin-1 is a strong prognostic indicator in stage II colonic cancer: a tissue microarray study. Mod Pathol 2005;18:511-518.

14 Kominsky SL, Agrani P, Korz D, et al. Loss of tight junction protein claudin-7 correlates with histological grade in both ductal carcinoma in situ and invasive ductal carcinoma of the breast. Oncogene 2003; 22:2021-2033.

15 Usami Y, Chiba H, Nakayama F, et al. Reduced expression of claudin-7 correlates with invasion and metastasis in squamous cell carcinoma of the esophagus. Hum Pathol 2006;37:569-577.

16 Swisshelm K, Macek R, Kubbies M. Role of claudins in carcinogenesis. Adv Drug Deliv Rev 2005;57:919-928.

17 Michl P, Barth C, Buchholz M, et al. Claudin-4 expression decreases invasiveness and metastatic potential of pancreatic cancer. Cancer Res 2003;63: 6265-6271.

18 Hough CD, Sherman-Baust CS, Pizer ES, et al. Largescale serial analysis of gene expression reveals genes differentially expressed in ovarian cancer. Cancer Res 2000;60:6281-6287.

19 Rangel LB, Agrawal R, D’Souza T, et al. Tight junction proteins claudin 3 and claudin 4 are frequently overexpressed in ovarian cancer but not in ovarian cystadenomas. Clin Cancer Res 2003;9:2567-2575.

20 Lee JW, Lee SJ, Seo J, et al. Increased expressions of claudin-1 and claudin-7 during the progression of cervical neoplasia. Gynecol Oncol 2005;97:53-59.

21 Hewitt KJ, Agarwal R, Morin PJ. The claudin gene family: expression in normal and neoplastic tissues. BMC Cancer 2006;6:186. 
22 Scopa CD. Histopathology of thyroid tumors. An overview. Hormones 2004;3:100-110.

23 Rosai J. Appendix C. Staging of cancer. In: Houston M (ed). Rosai and Ackerman's Surgical Pathology, 9th edn. Mosby: London, 2004, pp 2809-2810.

24 Lodi C, Szabó E, Holczbauer A, et al. Claudin 4 differentiates biliary tract cancers from hepatocellular carcinomas. Mod Pathol 2006;19:460-469.

25 Sobel G, Németh J, Kiss A, et al. Claudin 1 differentiates endometrioid and serous papillary endometrial adenocarcinoma. Gynecol Oncol 2006;103:591-598.

26 Cheung ST, Leung KL, Ip YC, et al. Claudin-10 expression level is associated with recurrence of primary hepatocellular carcinoma. Clin Cancer Res 2005;11:551-556.

27 Kerjaschki D, Krisch K, Sleyter UB, et al. The structure of tight junctions in human thyroid tumors. A systematic freeze-fracture study. Am J Pathol 1979;96:207-225.

28 Cochand-Priollet B, Raison D, Molinie V, et al. Altered gap and tight junctions in human thyroid oncocytic tumors: a study of 8 cases by freeze-fracture. Ultrastruct Pathol 1998;22:413-420.

29 Fluge O, Bruland O, Akslen LA, et al. Gene expression in poorly differentiated papillary thyroid carcinomas. Thyroid 2006;16:161-175.

30 Lee SK, Moon J, Park SW, et al. Loss of the tight junction protein claudin 4 correlates with histological growth-pattern and differentiation in advanced gastric adenocarcinomas. Oncol Rep 2005;13:193-199.

31 Rosai J. Thyroid gland. In: Houston M (ed). Rosai and Ackerman's Surgical Pathology, 9th edn. Mosby: London, 2004, pp 515-594.

32 Kovacs CS, Mase RM, Kovacks K, et al. Thyroid medullary carcinoma with thyreoglobulin immuno- reactivity in sporadic multiple endocrine neoplasia type 2- B. Cancer 1994;74:928-932.

33 Soler AP, Miller RD, Laughlin KV, et al. Increased tight junctional permeability is associated with the development of colon cancer. Carcinogenesis 1999;8: 1425-1431.

34 Furuse M, Fujimoto K, Sato N, et al. Overexpression of occludin, a tight junction-associated integral membrane protein, induces the formation of intercellular multilamellar bodies bearing tight junction-like structures. J Cell Sci 1996;109:429-435.

35 Matsuda M, Kubo A, Furuse M, et al. A peculiar internalization of claudins, tight junction-specific adhesion molecules, during intercellular movement of epithelial cells. J Cell Sci 2003;117:1247-1257.

36 Blackman B, Russell T, Nordeen S, et al. Claudin 7 expression and localization in the normal murine mammary gland and murine mammary tumors. Breast Cancer Res 2005;7:R248-R255.

37 Tobioka $\mathrm{H}$, Isomura $\mathrm{H}$, Kokai $\mathrm{Y}$, et al. Polarized distribution of carcinoembryonic antigen is associated with a tight junction molecule in human colorectal adenocarcinoma. J Pathol 2002;198:207-212.

38 Tobioka H, Tokunaga Y, Isomura H, et al. Expression of occludin, a tight-junction-associated protein, in human lung carcinomas. Virchows Arch 2004;445: 472-476.

39 Sheehan GM, Kallakury BVS, Sheehan CE, et al. Loss of claudins-1 and -7 and expression of claudins-3 and -4 correlate with prognostic variables in prostatic adenocarcinomas. Human Path 2007;28:564-569.

40 Saitou M, Fujimoto K, Doi Y, et al. Occludin-deficient embryonic stem cells can differentiate into polarized epithelial cells bearing tight junctions. J Cell Biol 1998;141:397-408. 